\title{
Implementation of efficient proactive computing using lazy evaluation in a learning management system
}

\author{
D. Zampuniéris* \\ CICeL, Faculty of Sciences, Technology \& Communication, University of Luxembourg, 6 rue Richard \\ Coudenhove-Kalergi, L-1359 Luxembourg-Kirchberg, Grand-Duchy of Luxembourg
}

In [1] we proposed a new kind of Learning Management Systems: proactive LMS, designed to help their users to better interact online by providing programmable, automatic and continuous analyses of users (inter)actions augmented with appropriate actions initiated by the LMS itself. The proactive part of our LMS is based on a dynamic rules-based system. But the main algorithm we proposed in order to implement the rules running system, suffers some efficiency problems. In this paper, we propose a new version of the main rules running algorithm that is based on lazy evaluation in order to avoid unnecessary and timecostly requests to the LMS database when a rule is not activated, that is: when its actions part will not be performed because preliminary checks failed.

Keywords learning management systems; proactive systems; intelligent tutoring systems; lazy evaluation

\section{Introduction}

Learning Management Systems (LMS), or e-learning platforms, are dedicated software tools intended to offer a virtual educational and/or training environment online. Despite a large number of functions covering a large number of users needs for a variety of different users acting specific roles in these environments, current LMS are fundamentally limited tools. Indeed, they are only reactive software, developed like classical, user-action oriented software. These tools wait for an instruction, most likely given through a graphical user interface, and then react to the user request.

One could imagine and hope for more helping and assisting tools, especially because:

1) users could be inexperienced online software users who would expect some guidelines (what to do and how to do it) from the system instead of a static user interface,

2) some particular users like e-tutors have to peruse lots of data in order to try to efficiently manage specific other users' needs and would expect some highlighting (where to search and what to look for) from the system instead of a static database.

In [1] we proposed a new kind of Learning Management Systems: proactive LMS, designed to help their users to better interact online by providing programmable, automatic and continuous analyses of users (inter)actions augmented with appropriate actions initiated by the LMS itself.

Proactive systems (see e.g., [2, 3]) adhere to two premises: working on behalf of, or pro, the user, and acting on their own initiative, without user's explicit command. Proactive behaviours are intended to cause changes, rather than just to react to changes. This is a major change from interactive computing, in which we lock a system into operating at exactly the same frequency as we do.

Our proactive LMS can automatically and continuously take care of e-students with respect to previously defined procedures rules, and even notify an e-tutor if something "wrong" is detected in an elearner behaviour; it can also automatically check some awaited behaviours of e-students, and react if these actions did not happen. Automatic and user-specific check of generic access conditions (prerequisites) to e-learning modules can be implemented using dynamic rules in the proactive system; finally, some automatic management processing of the LMS can also be performed by using the proactive part of the system.

\footnotetext{
* Corresponding author e-mail: denis.zampunieris@uni.lu
} 
The proactive part of our LMS is based on a dynamic rules-based system. But the algorithm we proposed in order to implement the rules running system, suffers some efficiency problems mainly due to lots of database requests when running the rules, some of them being superfluous.

In this paper, we propose a new version of the main rules running algorithm that is based on lazy evaluation in order to avoid unnecessary and time-costly requests to the LMS database when a rule is not activated, that is: when its actions part will not be performed because preliminary checks failed. In computer programming, lazy evaluation is a technique that attempts to delay computation of expressions until the results of the computation are known to be needed [4].

\section{Dynamic rules running system}

The set of rules is stored by the proactive system into a FIFO list: the oldest generated rule is at the beginning of the list and will be run first. Two parameters influence the behaviour of the rules running system: $\mathrm{F}=$ the time frequency of its activation periods, and $\mathrm{N}=$ the (maximum) number of rules it runs during an activation period. These parameters are set by the system manager and can be changed at runtime. The LMS activates (starts) the rules running system with respect to the parameter F. If the rules running system is already activated, it continues its current activation.

Once activated, the rules running system executes the $\mathrm{N}$ first rules of the FIFO list (if available), one at a time with respect to their ranks, using the algorithm shown at the end of this section.

Once run, a rule is discarded from the system. If one wants the rule (or more precisely: the proactive behaviour this rule implements) to stay active in the system for a longer time, the rule has to clone itself in order to be included in the next activation of the rules running system.

A rule is made of five parts: data acquisition, activation guards, conditions, actions and rules generation. These parts are successively briefly described hereunder. We will not enter into syntactic details.

The first part (data acquisition) allows a rule to get information from the LMS in order to use these data in its other parts. This implies that the data acquisition part is the first one to be performed when a rule is run. These data are stored into variables local to the rule; their values can not be modified by the rule - these are read only variables (but they can be used as references to access and modify values into the LMS database) and are discarded once the rule is run.

The second part (activation guards) is performed after the data acquisition part and is made of a set of AND-connected tests on local variables that, once evaluated, determine if the conditions and actions parts will be performed afterwards. Note that the last part of the rule, rules generation, is always performed. If all the activation guards are evaluated positively, then the conditions and actions parts are performed. On the contrary, these parts are ignored when running the rule. There is a special and automatically defined local Boolean variable called "activated" which value is set accordingly to the result of the guards evaluation.

The third part (conditions) is made of a set of AND-connected tests on local variables that, once evaluated, determine if the actions part will be performed afterwards. Syntax and semantics of conditions tests are equivalent to activation guards' tests.

The fourth part (actions) is made of a list of instructions that will be performed in sequence if all the conditions part tests are evaluated positively.

The fifth and last part (rules generation) is performed at the end. It allows the rule to generate other(s) rule(s) that will be performed afterwards. With this mechanism, one can program long-lasting rules that perform actions over a period of time.

The main algorithm to run a rule is as follows. This algorithm is written in pseudo-code and without low-level details for clarity purposes.

\footnotetext{
i. repeat for each data acquisition request DA a.perform DA

b.if error then raise exception on system manager console and go to step vii else create new local variable and initialize it with result of DA

ii.create new local Boolean variable "activated" initialized to false
} 


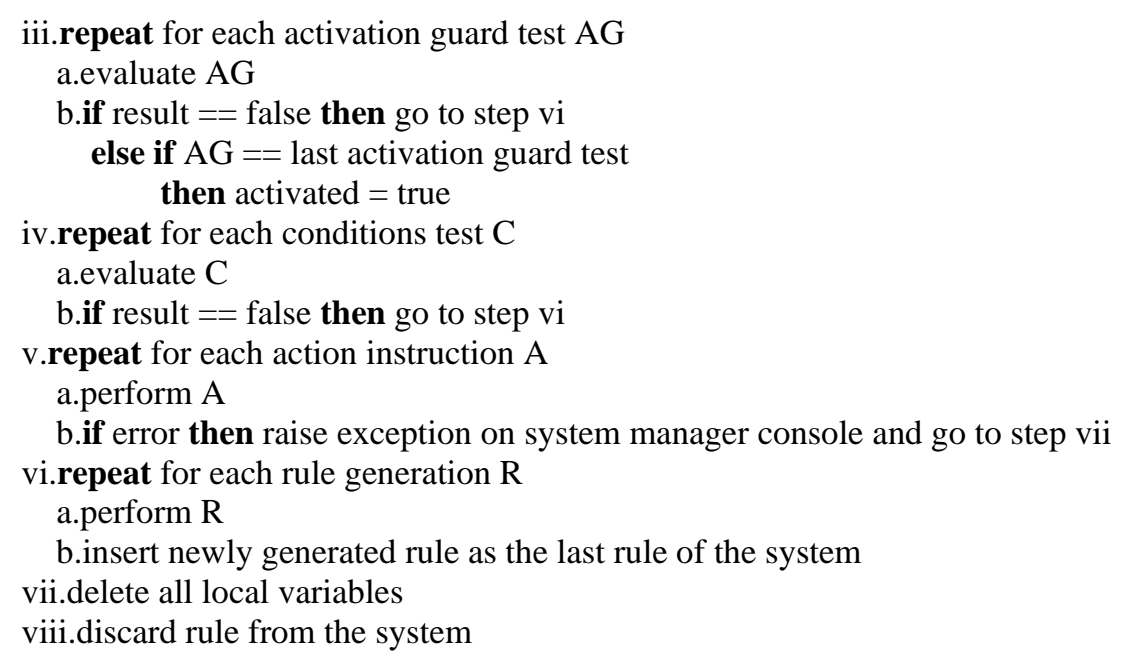

\section{Example of rules contents and uses}

Here follow the declarations of two rules that can be automatically added to the system when an estudent $(\mathrm{id}=\mathrm{S})$ is registered to an e-course $(\mathrm{id}=\mathrm{C}$ ) under the coaching of an e-tutor $(\mathrm{id}=\mathrm{T})$, even if these two rules will be activated only weeks later. This first example is intended to show how the proactive system can automatically take care of e-learners, and even notify an e-tutor if something "wrong” is detected in the e-learner behaviour.

The first rule is intended to give some welcome and recommendation words to the e-student, the first time she/he connects to the e-course:

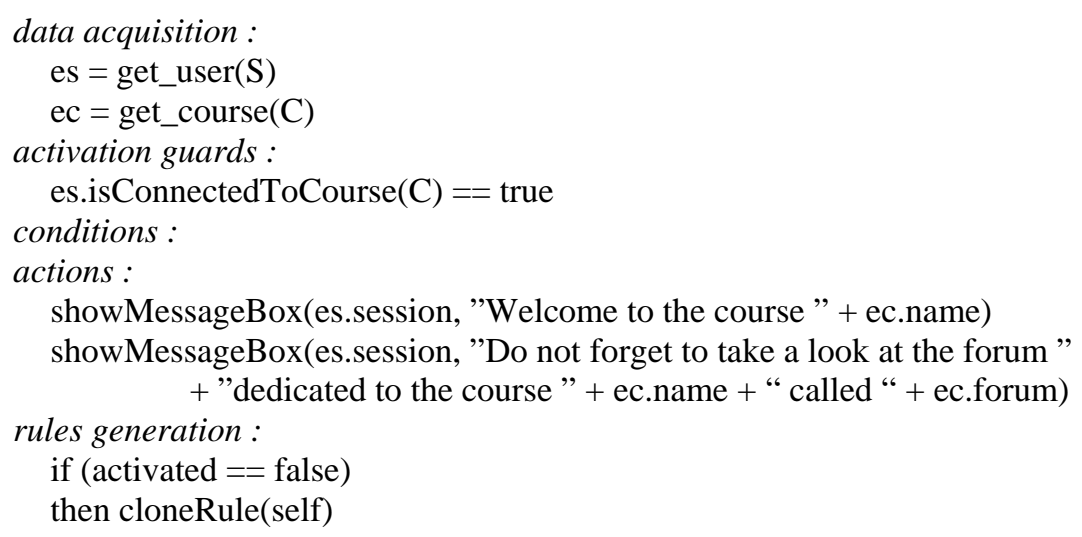

The second rule is intended to check that the same e-student S used at least one time the LMS forum communication tool dedicated to the e-course $\mathrm{C}$ one week after the start of that e-course and if not, to notify it by an LMS email to the e-tutor T so that she/he can check with her/him what is the problem.

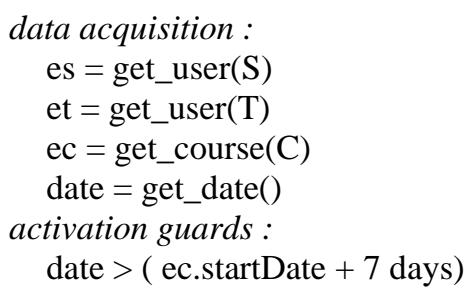




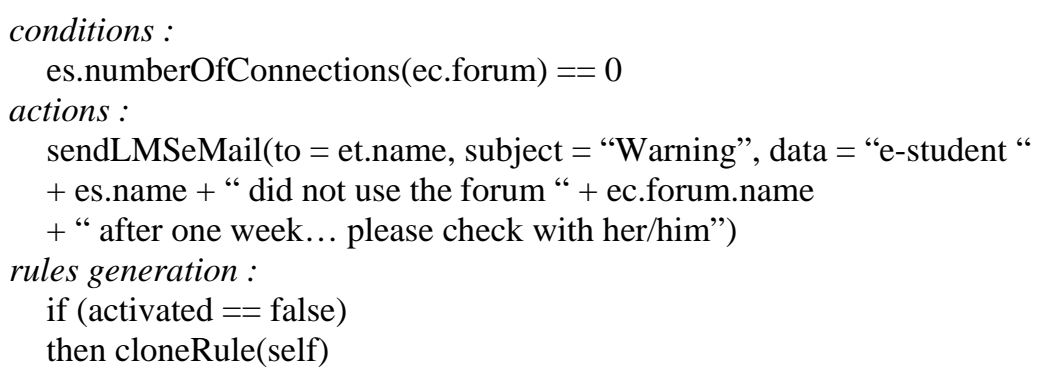

The second example gives a flavour of automatic management of the LMS by using the proactive system. The rule hereunder automatically collects the number of users connected to the LMS every 5 minutes, and stores it in a dedicated table in the LMS database, for later statistics purposes.

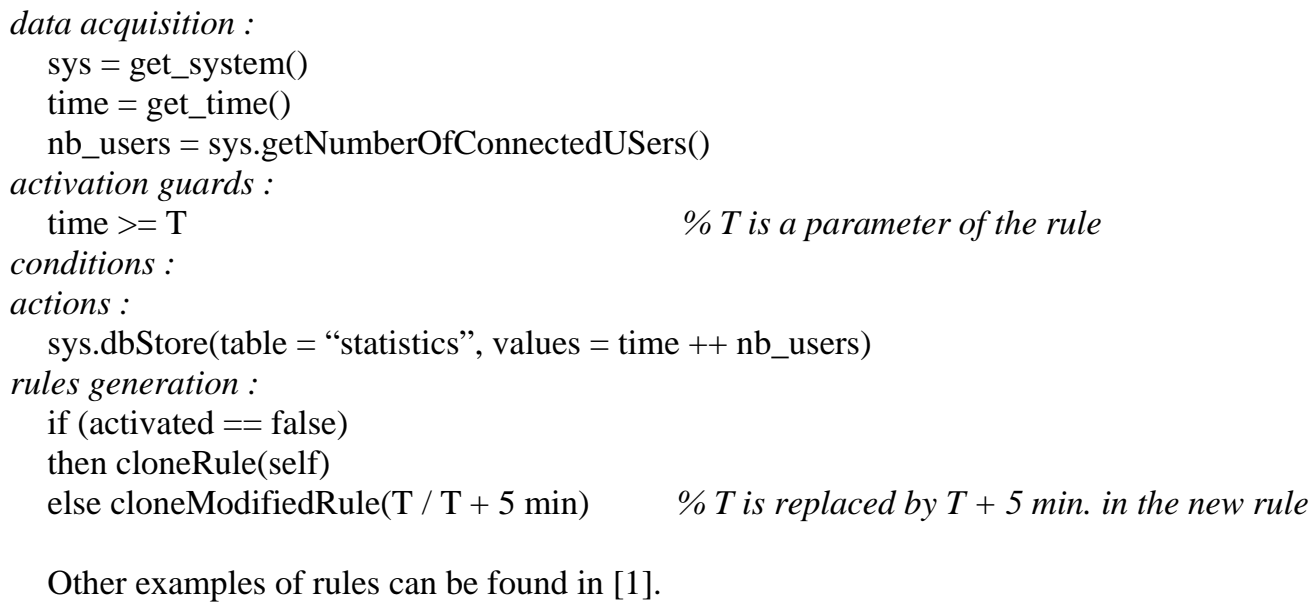

\section{Lazy evaluation of rules}

In the last example hereabove, for the sake of efficiency, the database request for the current number of connected users should be performed in the actions part, that is: only if the rule is activated. Indeed, if the rule is not activated (when the activation guard evaluated to false) there is no need to know this data. Hence the database request, which is time- and ressource-costly, is unuseful and should be avoided.

This efficiency problem might appear as minor in this example but becomes major with the continuous evaluation of large sets of rules. Indeed, as every rule makes an average of two to three requests to the LMS database, and as several desired proactive behaviours combined with a large number of targeted e-students and e-tutors result in a very large number of rules to continuously evaluate, the consequence is an even larger number of successive database requests in order to run the whole set of rules. During the processing of these database accesses, the mean reaction time of the LMS to a user request is increased, sometimes in a severe way.

This problem can be solved with the introduction of "lazy evaluation" in the main algorithm shown in chapter 2. In computer programming, lazy evaluation is a technique that attempts to delay computation of expressions until the results of the computation are known to be needed [4].

In our new LMS, lazy evaluation of rules in the proactive system is used in order to avoid unnecessary and time-costly requests to the LMS database when a rule is not activated, that is: when its actions part will not be performed because preliminary checks failed.

Technically speaking, a variable is no more composed of a pair <name, value $>$ but of a triple $<$ name, definition, value*> where definition is the expression that has been or will be evaluated in order to give a value to the variable, and value* is either a real value or the special value "to_be_computed". 
When a rule is run, in its data acquisition part, local variables to the rule are created but are not given a value; instead a triple $<$ name, definition, value ${ }^{*}>$ is generated for each variable, with its definition component equal to the expression to be computed and its value* component equal to the special value "to_be_computed".

When running the other parts of the rule, if the value of a variable is requested then either its value* component is equal to the special value "to_be_computed" or is a different one. In the first case, the expression attached to this variable, stored in its definition component, is computed and the result of this evaluation is stored into its value* component. This data is then usable as the value of the variable.

In the second case, it means that the expression attached to this variable in the data acquisition part of the rule has already been computed previously to give a value to the variable, and therefore this value can be directly used.

Back to the last example in the previous section (production of statistics), the sentence $<<$ nb_users $=$ sys.getNumberOfConnectedUSers() $>>$ in the data acquisition part will not result in a database request because the value of the local variable "nb_users" is not computed at that time. This database request will only be performed later when the value of the variable is requested: that is, when the sentence $<<$ sys.dbStore(table = "statistics", values = time ++ nb_users) $>>$ in the actions part of the rule is run.

\section{Conclusion}

Current Learning Management Systems (LMS) are fundamentally limited software tools: they are only reactive, user-action oriented software. These tools wait for an instruction, most likely given through a graphical user interface, and then react to the user request.

In [1] we proposed a new kind of Learning Management Systems: proactive LMS, designed to help their users to better interact online by providing programmable, automatic and continuous analyses of users (inter)actions augmented with appropriate actions initiated by the LMS itself. The proactive LMS can automatically and continuously take care of e-students with respect to previously defined procedures rules, and even notify an e-tutor if something "wrong” is detected in an e-learner behaviour; it can also automatically check some awaited behaviours of e-students, and react if these actions did not happen.

The proactive part of our LMS is based on a dynamic rules-based system. But the main algorithm we proposed in order to implement the rules running system, suffered some efficiency problems resulting in an increase of the mean reaction time of the LMS to a user request, sometimes in a severe way.

In this paper, we proposed a new version of the main rules running algorithm that is based on lazy evaluation in order to avoid unnecessary and time-costly requests to the LMS database when a rule is not activated, that is: when its actions part will not be performed because preliminary checks failed. When using such a delayed evaluation mechanism, an expression is not evaluated as soon as it gets bound to a variable, but when the evaluator is forced to produce the expression's value.

Future work include the design and the implementation of sets of rules (packages) dedicated to common users needs that one will be able to use "as is", as well as "abstract” packages (templates), that one will have to tailor to specific users needs by using appropriate tools.

\section{References}

[1] D. Zampunieris, "Implementation of a proactive learning management system", Proceedings of E-Learn World Conference on E-Learning in Corporate, Government, Healthcare, \& Higher Education, Hawaii, USA, October 2006, Eds. Th. C. Reeves and Sh. F. Yamashita, ISBN 1-880094-60-6.

[2] D. Tennenhouse, "Proactive computing", Communications of the ACM, 43, 5 (2000), pp. 43-50.

[3] A. Salovaara and A. Oulasvirta, "Six modes of proactive resource management: A user-centric typology for proactive behaviors", Proceedings of the Nordic conference on human-computer interaction, ACM international conference proceedings series, 82 (2004), pp. 57-60.

[4] Wikipedia, the free encyclopedia, http://en.wikipedia.org/wiki/Lazy_evaluation . 